\title{
Case-Based Reasoning Systems: From Automation to Decision-Aiding and Stimulation
}

\author{
Soumitra Dutta, Member, IEEE, Berend Wierenga, and Arco Dalebout
}

\begin{abstract}
Over the past decade, case-based reasoning (CBR) has emerged as a major research area within the artificial intelligence research field due to both its widespread usage by humans and its appeal as a methodology for building intelligent systems. Conventional CBR systems have been largely designed as automated problem-solvers for producing a solution to a given problem by adapting the solution to a similar, previously solved problem. Such systems have had limited success in real-world applications. More recently, there has been a search for new paradigms and directions for increasing the utility of CBR systems for decision support. This paper focuses on the synergism between the research areas of CBR and decision support systems (DSSs). A conceptual framework for DSSs is presented and used to develop a taxonomy of three different types of CBR systems: 1) conventional, 2) decision-aiding, and 3) stimulative. The major characteristics of each type of CBR system are explained with a particular focus on decision-aiding and stimulative CBR systems. The research implications of the evolution in the design of CBR systems from automation toward decision-aiding and stimulation are also explored.
\end{abstract}

Index Terms-Decision support systems, knowledge-based systems, case-based reasoning, case-based decision support systems, intelligent decision support systems, intelligent agents.

\section{INTRODUCTION}

I NTERACTIONS between the fields of decision support systems (DSSs) [22], [32], [55] and artificial intelligence (AI) [11], [51] have proven to be a fruitful area for research over the last years. With the help of AI techniques DSSs have incorporated the heuristic models of decision-makers (DMs) and provided increasingly richer support for decisionmaking. AI systems have also benefited from DSS research as they have scaled down their goal from replacing to supporting DMs. While most of the attention in the literature has focused on the integration of expert systems and DSSs [46], [60], this research focuses on the synergies between case-based reasoning (CBR) [24], [36], [52], [56] and DSSs.

Simply described, CBR is concerned with creating solutions for problems by adapting solutions to previously solved problems. CBR has emerged as a major research area within AI over the last decade due to its widespread usage by humans and its appeal as a methodology for building intelligent systems. CBR systems described in the literature typically behave like automated problem-solversproducing "solutions" to a problem by retrieving and adapting a prior solution. Such systems have often had limited success in real world applications due to both a frequent scarcity of appropriate cases and the complexity of case adaptation procedures which have largely defied generalization and standard solution routines.

More recently, CBR researchers have started exploring new paradigms and directions for increasing the utility

- S. Dutta is with European Institute of Business Administration (INSEAD), Fontainebleau, France 77305. E-mail: dutta@insead.fr.

- B. Wierenga and A. Dalebout are with the Rotterdam School of Management, Department of Marketing, Erasmus University, Rotterdam, Holland 3000 DR. E-mail: \{b.wierenga, a.dalebout\}@fac.fbk.eur.nl.

Manuscript received 21 July 1995.

For information on obtaining reprints of this article, please send e-mail to: tkde@computer.org, and reference IEEECS Log Number 104432. of CBR systems. For example, the idea of using CBR for decision-aiding has been proposed in the literature [10], [36], [38]. Using CBR systems for decision-aiding has a certain intuitive appeal because such man-machine collaboration in decision-making can be mutually beneficial. A CBR system can help overcome some of the limitations of casebased reasoning by humans. For example, it can augment a decision-maker's (DM's) memory by providing access to a large collection of cases, rapidly recalling the most relevant cases, and aiding the decision process through appropriate critiquing. In return, the DM can assume greater responsibility for the use of cases, i.e., the adaptation of prior cases to the current decision. While humans have been observed to be fairly good at adapting cases, these adaptation procedures have proven to be the Achilles heel for many CBR systems [38].

Another new paradigm which is generating interest is the use of CBR systems to stimulate innovative decisionmaking and enhance learning about the decision situation in a DM [1]. Such learning oriented CBR systems can be viewed as symbiotic systems in which the human DM and the CBR component play mutually complementary stimulative roles in the decision-making process. By exploiting prior problem solving experiences stored within cases in the case library, CBR can provide the opportunity to emphasize the creative divergent aspects of learning such as metaphorical thinking and lateral stimulation.

Prior research in such new paradigms for CBR systems has been limited and has lacked adequate conceptual bases. This paper augments prior research in CBR systems by using an integrative conceptual framework for the design of DSSs to classify CBR systems into three categories: conventional, decision-aiding and stimulative. Conventional CBR systems are outcome oriented and tend to behave like traditional automated problem-solvers. Decision-aiding CBR 
systems are process oriented and tend to emphasize supporting the process of decision-making by a DM. Stimulative CBR systems are learning oriented and aim to enhance learning in the DM about the decision situation and the decision process. Each type of a CBR system is suited to a particular organizational decision environment and CBR systems can be seen to be evolving from conventional to decision-aiding and stimulative in nature. The desired nature of the CBR system has important implications for the design of the system.

The structure of the remainder of this paper is as follows. Section 2 introduces selected important developments in the field of DSSs and presents an integrative framework for the design of DSSs. Next, this framework is used to identify three different categories of CBR systems. This classification of CBR systems and its implications for decision support are the subject of Section 3. Section 4 outlines the major characteristics of each type of CBR system with brief descriptions of representative examples. The final section concludes the paper by highlighting the contributions of this paper and describing avenues for further research.

\section{DeCision SupPort SyStems}

\subsection{Introduction}

Decision support systems are interactive, computer-based systems helping decision-makers (individuals and/or groups) to solve various semistructured and unstructured problems involving multiple attributes, objectives, and goals. Historically, the majority of DSS have been either computer implementations of mathematical models (e.g., optimization algorithms) or extensions of database systems and traditional management information systems. More recently, researchers [2], [45], [48], [61] have argued that DSS should focus on defining flexible learning-oriented environments to support decision-makers (DMs) in recursively redefining their problems, trying out different solution possibilities and eventually reaching a state of cognitive equilibrium [61]. Such an emphasis on learning has led to a different type of "learning-oriented" DSS [2], [26], [61] to support DMs in incrementally exploring a decision situation while avoiding typical decision-making biases observable in practice [29].

\subsection{A Conceptual Framework for Decision Support Systems}

Several frameworks for DSS have been proposed in the literature and they focus on either the technological platforms underlying the DSS [6], [8], or the activities related to the development of DSS [16], [30], [47], [58], or the nature of decision support provided by the DSS [13], [22], [23], [44] or a combination of the above three perspectives [3], [17], [55], [57].

The conceptual framework used in this research provides an integrative perspective for the design of DSSs. The framework used in this paper integrates three different objects (outcome, process, and learning) of decision support with three modes (automate, informate, and stimulate) of decision support to yield significant DSS design characteristics (restrictiveness, guidance, and customizability). The framework is briefly described below. More details on the framework and extended comparisons with other conceptual frameworks in the DSSs literature are provided in reference [13].

\subsubsection{Object of Decision Support}

The object of decision support is concerned with the question: what to support? Three different objects of decision support are emphasized in the framework: outcome, process and learning.

The outcome support view of decision-making is primarily concerned with the final decision. The relevant question in this context is: what is the decision? The emphasis is on ensuring that the best or "correct" output is produced for an appropriate set of inputs. The process used to transform the inputs into the outputs is not of primary concern.

In the process support view of decision-making, the emphasis is on the process by which decisions are made, and not solely on the final outcome. The relevant question in this context is: How was the decision reached?

When the object of decision support is learning, the relevant question is: how to improve the decision process? The emphasis is on the ability to question decision procedures and adopt new innovative decision processes with the ultimate aim of improving the decision.

\subsubsection{Mode of Decision Support}

The mode of decision support is concerned with the question: How to provide support? Three different modes of decision support are considered in the framework: automate, informate, and stimulate.

Automation of decision-making has been the strength of traditional DSSs. DSSs which use the automate mode of decision support tend to generate "solutions" by solving models or "hard-coded" solution procedures.

In the informate mode of decision support, the emphasis is not so much on generating a solution by solving a model or a "hard-coded" solution, but rather on informing and guiding DMs through the different phases of decisionmaking. Knowledge about the process of arriving at a decision can often be richer and more useful than the final decision itself as it supports a DM's creative thinking in determining the solution [1].

The final mode of decision support is the ability of DSSs to stimulate DMs into new and innovative decision procedures. Proper stimulation can aid DMs to notice special patterns in the decision environment, explore new solution designs, test alternative hypotheses, and reflect on the obtained results.

\subsubsection{System Design Dimensions}

The system design of a DSS has to be related to the object and mode of decision support required from the DSS. The relevant question along this dimension is: What is the impact of the DSS design on the decision support requirements? The framework uses the three system design dimensions identified by Silver [55]: restrictiveness, guidance, and customizability.

While restrictiveness has been described [55] as "the degree to which, and the manner in which, a DSS limits 
its user's decision-making processes to a subset of all possible processes," guidance is defined as "... subject to what users can do, how the system affects what they do." Highly restrictive systems limit the degree of decisional guidance possible and vice versa. Customizability refers to the degree to which DMs are able to adapt and specialize DSSs to fit the special characteristics of their respective decision situations.

\subsubsection{The Framework}

The framework maps the system design dimensions onto the dominant matches between the objects and modes of decision support. Highly restrictive DSSs are best suited to the outcome object and automate mode of decision support as they tend to limit choice. If the primary organizational consideration is that a particular outcome is reached, then highly automated and restrictive DSSs are appropriate.

Guidance oriented DSSs are well adapted to the process object and informate mode of decision support. If the process by which decisions are made is of concern to the organization, then it is important to design DSSs which are able to provide high degrees of decisional guidance to decision-makers and informate them about "how to" navigate through the decision space.

The learning view of decision-making calls for greater emphases on stimulation and customizability in DSSs. If the organization wants to learn and innovate continuously in its decisions, then it is important to design DSSs which stimulate DMs into new exploratory modes of problem solution and allow them to continuously adapt the DSS to the changing decision needs of the environment.

Note that the framework presented above is not centered on the task or problem to be tackled by the DSS. This research takes the position that given a particular task/ problem, the factors affecting DSS design are more related to the object and mode of decision support rather than the task/problem itself. This is because the same task/problem can be tackled by different foci on the objects and modes of decision support. Depending upon the required relative emphases of the objects and modes of decision support, different requirements are imposed on DSS design. For example, a DSS for medical diagnosis may be designed in an outcome/automate approach for naive users. An alternate DSS for the same medical diagnosis problem may be designed in the process/informate approach for more experienced users.

\section{Classifying CBR Systems}

Viewed in the context of the conceptual framework of Fig. 1, it is possible to identify three different categories of CBR systems:

1) conventional,

2) decision-aiding, and

3) stimulative.

These types of CBR systems are described in more detail below.

\subsection{Conventional CBR Systems}

The objective of conventional CBR systems is similar to most other AI systems: To automate problem solution. Simply described, conventional CBR systems automate the retrieval of a case from the case library and its adaptation to the current problem to generate a solution. The DM does not play a significant role in both the case retrieval and adaptation procedures and only acts at the very end to either accept or reject the solution produced by the CBR system.

Most CBR systems described in the literature are conventional in nature. For example, CHEF [24], [25] uses a case library of prior recipes to produce a feasible recipe for a certain problem description and MEDIATOR [37] uses prior cases to suggest a solution for disputed resources.

Implicit in the tendency to automate within conventional CBR systems is an emphasis on the decision outcome over the decision process, on providing solutions rather than facilitating problem solution and on the system over the DM. Such systems also tend to be fairly restrictive as their adaptation skills arise from possessing structured knowledge of a limited domain.

Viewed against the framework of Fig. 1, conventional CBR systems can be positioned at the bottom left corner favoring the outcome object of decision-making with a high degree of automation and restrictiveness. This is depicted in Fig. 2.

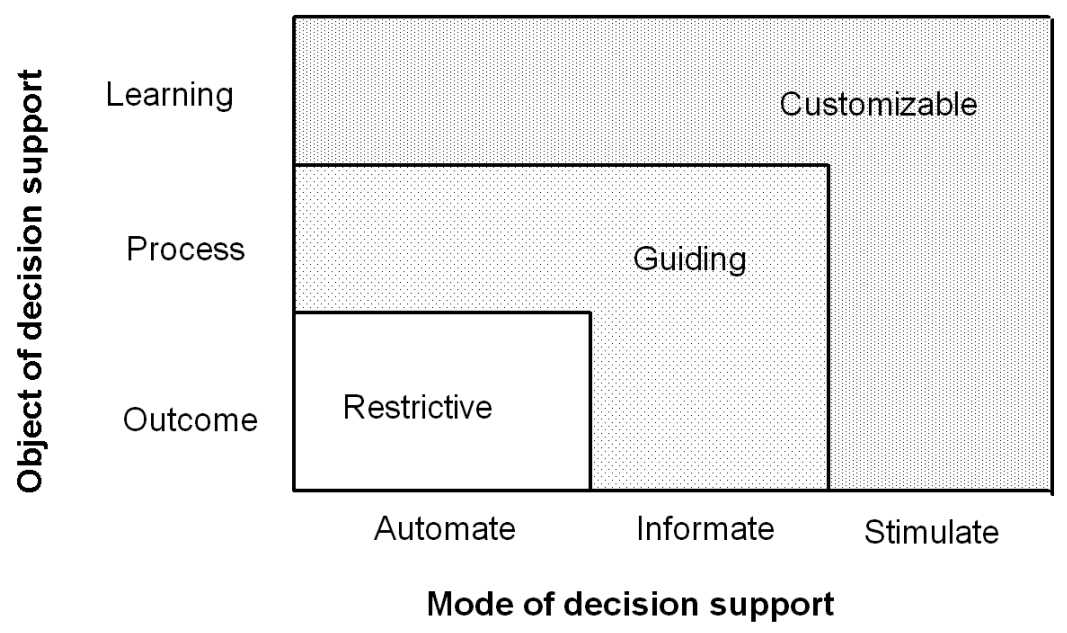

Fig. 1. Conceptual framework. 


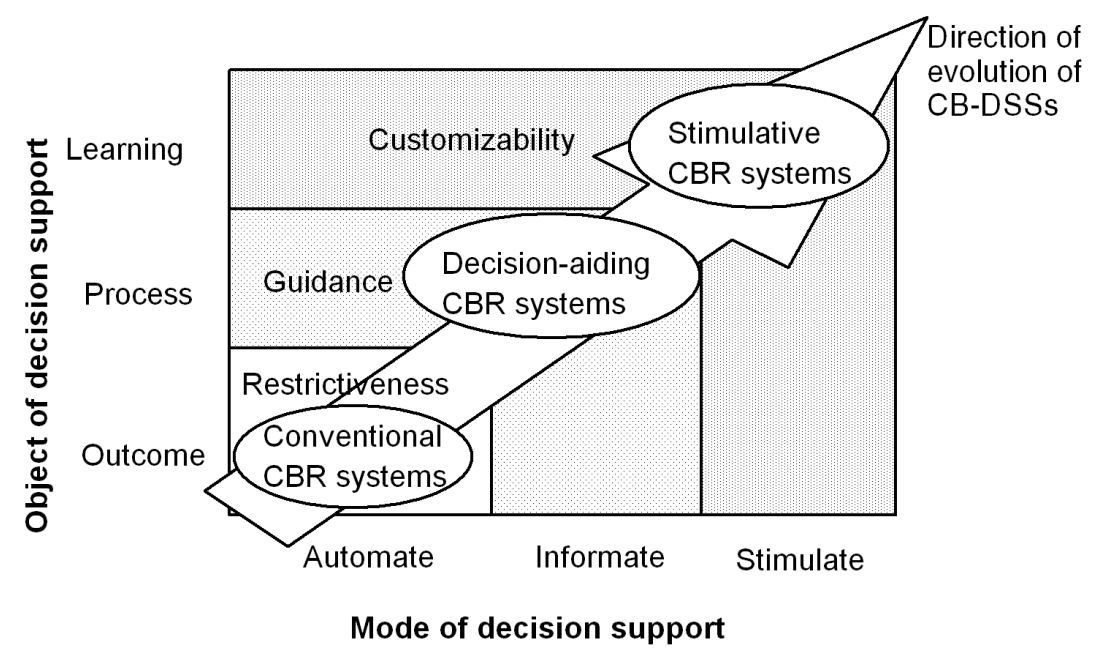

Fig. 2. Categories of case-based reasoning systems.

\subsection{Decision-Aiding CBR Systems}

While humans commonly use CBR, they have been observed to suffer [36] from an inability to consistently recall the appropriate set of prior cases, distinguish between important and unimportant features, recall prior experiences under time pressures, and deal with incomplete and uncertain information in current problems. These limitations are more acute for novices who lack a sufficiently complete (and large) collection of prior experiences.

Decision-aiding CBR systems aim to support a DM in taking a decision. Rather than automating all steps of the case-based reasoning cycle as is common in conventional CBR systems, Kolodner [36, p. 65] has suggested that it may be mutually beneficial to design CBR systems for aiding decision-making: "Because people are good at using cases but not as good at recalling the right ones, useful systems could be built that augment human memory by providing people with cases that might help them to reason but allowing all the complex reasoning and decision-making to be done by the person."

Kolodner [36], [38] has classified the support offered by decision-aiding CBR systems as either "active" or "passive." Passive support is defined to be similar to a smart database interface with the ability to retrieve cases based on partial matches and answer specific questions on the retrieved case. Active support is defined as the ability to retrieve cases, warn of potential problems, and help in critiquing, with the DM being responsible for adaptation procedures, choice of features and cases to consider, and the evaluation of suggestions and warnings. Given the complexity of the adaptation process in conventional CBR, it has been suggested [36], [38] that it is best to let the DM take the lead in adapting prior cases and make the final decision regarding the current problem.

Note that the move from conventional automation to decision-aiding represents an important shift in the design and use of CBR systems. CBR is no longer required to simply generate a solution, but is viewed as facilitating the process of decision-making or as making "interventions" [55] in the decision process. This implies a greater stress on the process object of decision support with corresponding emphases on the informate and guidance aspects of the mode of decision support and system design dimensions respectively. Thus decision-aiding CBR systems can be positioned in the middle of our framework as shown in Fig. 2.

A small number of CBR systems from the literature can be described as decision-aiding in nature. Most such systems [20], [27] act as smart retrieval systems and aim to provide an interactive interface to the DM via which the DM can control some aspects of the case retrieval or case adaptation procedures. Some recent decision-aiding CBR systems [1], [12] go beyond this and focus on capturing and exploiting the decision process of the DM. More details on these systems are provided in the next section.

\subsection{Stimulative CBR Systems}

The importance of DSSs stimulating DMs and actively enhancing learning has been recognized in the literature [2], [45], [48], [61]. These ideas are of central concern in stimulative CBR systems. Such systems aim not merely to aid decision-making but rather to actively encourage learning through reflective observation in the DM. Experiential learning theory [35] provides a useful theoretical construct to relate $\mathrm{CBR}$ and learning.

Experience plays a central role in experiential learning theory in which learning is conceived as a continuous process of adaptation between four states: concrete experience, reflective observation, abstract conceptualization and active experimentation. The phenomenon of learning occurs as a DM resolves and balances the tensions and conflicts between the convergent (abstract conceptualization and active experimentation) and divergent (concrete experience and reflective observation) modes of adaptation.

Prior research in decision-making and DSSs have been convergent in nature as witnessed by the extensive research on the rational evaluation of solution alternatives [35]. The integration of experience as cases in CBR within DSSs provides the opportunity to emphasize the creative divergent aspects of learning such as tolerance for ambiguity, metaphorical thinking and lateral stimulation, and create divergent DSSs. Viewed against our framework of Fig. 1, stimu- 
lative CBR systems can be positioned at the top right corner (see Fig. 2).

Few CBR systems can be truly classified as stimulative in nature. A system which comes close is a CBR agent [1] which dynamically influences the decision-making process by assuming the dual roles of an adviser and a storyteller. In the storyteller mode, the CBR agent retrieves and tells a "story" of the prior case with the aim to stimulate lateral thinking and reflective observations in the DM. In the adviser mode, the $C B R$ agent proactively interacts with the $\mathrm{DM}$ to stimulate his/her decision process.

Table 1 summarizes the distinctive features of the three types of CBR systems described above in light of the conceptual framework for DSSs depicted in Fig. 1. Table 2 classifies some well known CBR systems from the literature according to the three categories of case-based DSSs identified above.

It is evident from Table 2 that most CBR systems described in the literature are conventional in nature. Decision-aiding CBR systems are currently being researched actively and their number is steadily increasing. Enhancing learning in DMs has not been a major focus thus far within the CBR community.

CBR systems are today in the process of making the move from automated problem-solvers to decision-aiding systems. The next challenge is to design stimulative CBR systems. Referring to the classification of Fig. 2, the overall trend in the integration of CBR and DSSs is in moving from the lower left corner to the upper right corner. Increasingly, CBR is being used as a tool to informate and stimulate DMs with an emphasis on the process and learning objects of decision support.

\subsection{Decision Environments for CBR Systems}

All three types of CBR systems described above can be used for decision support and can thus be included under the generic label, case-based decision support system (CB-DSS). However the type of decision support provided by each type of CB-DSS varies and is better suited to a particular set of decision conditions.

Conventional CB-DSSs are designed to generate "automated" solutions. Thus they are intuitively more use-

TABLE 1

DifFERENT TYPES OF CASE-BASEd REASONING SYSTEMS

\begin{tabular}{|l|l|l|l|}
\cline { 2 - 4 } \multicolumn{1}{c|}{} & Conventional & Decision Aiding & Stimulative \\
\hline $\begin{array}{l}\text { Object of Decision } \\
\text { Support }\end{array}$ & Outcome & Process & Learning \\
\hline $\begin{array}{l}\text { Mode of Decision } \\
\text { Support }\end{array}$ & Automate & Informate & Stimulate \\
\hline $\begin{array}{l}\text { System Design } \\
\text { Characteristic }\end{array}$ & Restrictiveness & Guidance & Customizability \\
\hline
\end{tabular}

TABLE 2

CLASSIFICATION OF CASE-BASEd REASONING SYSTEMS FROM THE LITERATURE

\begin{tabular}{|c|c|c|}
\hline CB-DSS Type & Examples & Domain/Task \\
\hline Conventional & $\begin{array}{l}- \text { CHEF [24], [25] } \\
\text { - CASEY [40] } \\
\text { - JULIA [28] } \\
\text { - KRITIK [19] } \\
\text { - MEDIATOR [37] } \\
\text { - PERSUADOR [59] } \\
\text { PRIAR [31] } \\
\text { - } \text { ROUSM [21] } \\
\text { - } \text { SWALER [18] } \\
\text { SWA, [43] }\end{array}$ & $\begin{array}{l}\text { - Recipe creation } \\
\text { - Heart failure diagnosis } \\
\text { - Meal planning } \\
\text { - Physical devices } \\
\text { - Mediation } \\
\text { - Labor negotiations } \\
\text { - Planning in blocks world } \\
\text { - Inter-bank financial telexes } \\
\text { - Robot navigation } \\
\text { - Explaining anomalous events }\end{array}$ \\
\hline Decision-aiding & $\begin{array}{l}\text { - Battle Planner [20] } \\
\text { - CLAVIER [27] } \\
\text { - ARCHIE-2 [7] } \\
\text { - CAL [12] }\end{array}$ & $\begin{array}{l}\text { - Land warfare battle planning } \\
\text { - Autoclave loading } \\
\text { - Architectural design } \\
\text { - Marketing (Product manage- } \\
\text { ment) }\end{array}$ \\
\hline Stimulative & • MCDM-CBR [1] & - Multicriteria decision-making \\
\hline
\end{tabular}


ful when there is a high degree of structure in the decision problem and a relatively low level of uncertainty in the decision environment. In contrast, decision-aiding CB-DSSs aim to support rather than lead the decision processes of a DM. Thus they are potentially more useful in environments with lower levels of structure and higher levels of uncertainty. With increased information about the decision process, DMs can flexibly and meaningfully change critical parts of decision processes to respond to changes in the decision environment.

Though learning is possible in all decision situations, stimulative CB-DSSs probably have the most value for unstructured problems in dynamic decision environments. It is also important to have skilled DMs and an organizational decision environment which is flexible and encourages innovation. As a note of caution, one should be aware that decision-aiding and stimulative CBR-DSSs are relatively new, and more empirical research is needed to validate the conditions best suited to each type of CB-DSSs.

\section{Case-Based Reasoning in CB-DSSs}

This section outlines the major characteristics of CBR in each category of CB-DSSs with brief descriptions of representative examples.

\subsection{CBR in Conventional CB-DSSs}

Conceptually, the core of CBR in conventional CB-DSSs consists of matching the current problem to a store of cases to retrieve the most relevant similar case and then adapting the retrieved case to the current problem. This is depicted in Fig. 3. Many systems also store the final solution back in the case memory (to avoid duplication of effort in the future) as indicated by the dotted line in Fig. 3.

The major components of a generic case in a conventional CB-DSS are: the initial problem/situation description, the solution to the problem specified in the problem description and the outcome, i.e., the resulting state of the world after the stated solution was carried out. Case retrieval procedures typically match the current problem description with the initial problem descriptions in cases to retrieve the most similar (and relevant) case. Specialized knowledge, both commonsense and causal, about the domain of application of the CB-DSS is used to assist in the adaptation of solutions from prior cases.

Conventional CB-DSSs in the literature have adapted the reasoning procedure of Fig. 3 in different ways. For exam- ple, CHEF's [24], [25] architecture includes an "Anticipator" module to anticipate the goals of the automated planner and a special "Repairer" module to explain failures in the execution of solutions generated using CBR. Another system, Julia [28] includes a modified truth maintenance system [9] to ensure the internal consistency of solutions generated using CBR.

Regardless of their individual sophistication and mutual differences, conventional CB-DSSs largely act as automated problem-solvers. Because conventional CB-DSSs produce the solution for a "passive" user, they need to contain all knowledge, both commonsense and causal, required to solve the problem. Such knowledge is generally limited and while granting conventional CB-DSSs the ability to autonomously adapt and propose solutions in a specific domain, restricts their applicability across domains.

\subsection{CBR in Decision-Aiding CB-DSSs}

A small number of CB-DSSs can be described as decisionaiding in nature. Most such systems act as smart retrieval systems and aim to provide an interactive interface to the $\mathrm{DM}$ via which the DM can control some aspects of the case retrieval or case adaptation procedures.

For example, DMs using Battle Planner [20] are required to describe a battle situation and a solution plan to the system. The system uses the battle situation description and the proposed solution to retrieve similar cases. These cases along with case summaries are then used by the DM to critique his/her own solution. This leads to the DM proposing a modified solution for the battle description which is again used by the system to retrieve a new set of similar cases. This iterative procedure is carried out till the DM does not find the retrieved cases to be of additional value.

What is ignored and/or lacking in Battle Planner and similar systems is knowledge about the process by which the solution to the problem was reached. Presenting an analysis of why a solution works for a particular problem is different from understanding how the solution was reached. For most real world problems, the process of arriving at solutions is a nontrivial procedure consisting of several temporally separated, but interdependent decisions. Knowledge about the process of arriving at a decision can often be richer and more useful than the final solution itself as it encodes the creative thinking and experimentation that went into the determination of the solution.

Thus, more recent decision-aiding CB-DSSs have begun to explicitly focus on capturing and exploiting the decision

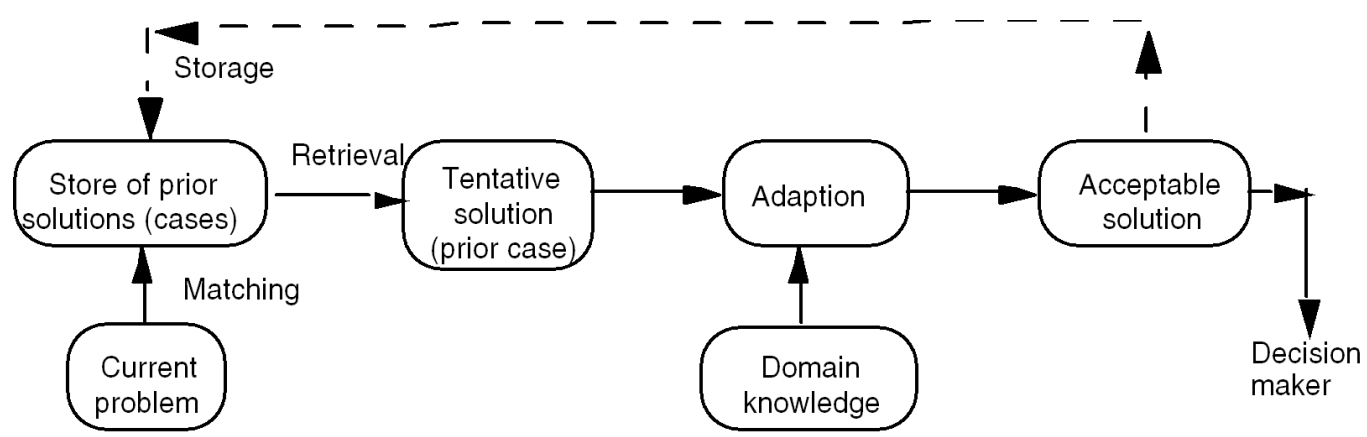

Fig. 3. Conventional case-based reasoning process. 
processes of DMs. For example, CAL [12], [13] is a process oriented decision-aiding CB-DSS which consists of the following four modules (see Fig. 4):

- Case memory: This acts as the repository of experience and stores decision-making processes as cases.

- Monitor: This module is responsible for both storing the current decision process as a case in the case memory and for monitoring the progress of the current decision process to determine conditions calling for interventions for decision-aiding.

- Analyzer: This component analyzes requests for advice from either the DM or the monitor module and retrieves appropriate cases from the case memory.

- Adviser: This module is responsible for advising the $\mathrm{DM}$ regarding the current decision process and problem solution.

It is interesting to compare the CBR methodology of CAL (Fig. 4) with that of a conventional CB-DSS (Fig. 3). A first observation is that CAL does not follow the "linear" solution methodology of conventional CB-DSSs: match-retrieve-adapt. CAL's solution methodology represents a more iterative cycle of monitor-analyze-advise. CAL does not emphasize the generation of solutions as much as intervening (advising) in the decision process at appropriate moments to aid the DM.

In addition to initial problem and final solution descriptions, cases in CAL explicitly capture the decision process used to obtain the solution. A decision-making process in CAL is described by a directed graph in which nodes represent descriptions of the decision process at particular instants of time and arcs represent transitions from one decision state to the next. Transitions between decision states can be caused either by events in the external world (not caused by the DM) or by specific actions undertaken by the DM with regard to the decision situation.

The Monitor module uses heuristics to detect decision intervention conditions, i.e., stages in the decision process at which the DM would benefit from guidance regarding the decision process. Examples of decision intervention conditions detected by the Monitor module are immobility (lack of progress by the DM in the decision process), irrelevance (possibly irrelevant actions by the DM during the decision process) and cyclicity (cyclical actions by the DM).
The Adviser module exploits process knowledge within cases to aid decision-making by the DM. In particular, it utilizes knowledge about the different states and transitions across states in prior decision processes to provide specific (current) decision process-related suggestions to the DM. The suggestions and advice offered by the Adviser module is related to the nature of the intervention condition identified by the Monitor module. The Adviser module does not attempt to produce the solution for the DM. Rather, it makes comparative suggestions about specific aspects of the current and prior decision processes.

\subsection{CBR in Stimulative CB-DSSs}

Stimulative CB-DSSs aim to enhance learning in the DM about the decision situation and the decision process. In general, learning-oriented DSS can be viewed [1] as symbiotic systems in which the human DM and the computerbased system play complementary and mutually supportive roles in the decision-making process. It is through their mutual interactions that learning about a specific decision takes place, with the system providing a continuous stimulus for the DM to become aware of and model explicitly his/her mental models through the exploration of different dimensions of the decision situation at hand.

Angehrn and Dutta [1] have noted that in learningoriented DSSs, the role played by the human component consists primarily in providing:

1) the context for the interaction, i.e., the initial problem perception;

2) the motivation for trying to explicitly represent and explore the given decision situation; and

3) the judgment driving this exploratory process.

On the other hand, the role of the computer-based system can be reduced to the following two components:

1) a facilitation component, i.e., a set of integrated tools enabling DMs to incrementally map their mental models into explicit representations which can be revised, analyzed, and explored [2]; and

2) a stimulus component, i.e., a set of tools which actively enhance learning and understanding throughout the decision-making process [2], [45], [48].

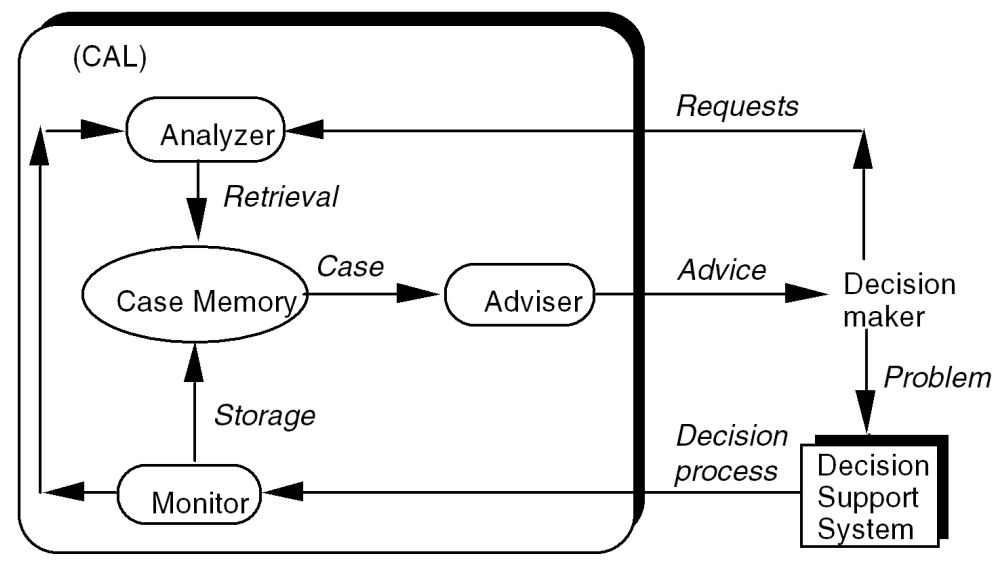

Fig. 4. Case-based reasoning in CAL, a decision-aiding case-based decision support system. 
Few CBR systems can be truly classified as stimulative in nature. Enhancing learning has not been a focus for CBR researchers and the first systems in this direction are only now being designed. For example, a CBR agent codesigned by one of the authors [1] for the class of multicriteria decision-making (MCDM) problems [14], [39] dynamically stimulates the decision-making process by assuming the dual roles of an adviser and a storyteller (see Fig. 5).

In the storyteller role, the CBR agent helps DMs in gaining alternative perspectives on the problem at hand by intervening autonomously during a decision-making session and exposing them interactively to how problems in different, but relevant contexts have been approached and solved. The main function of the storyteller role is to stimulate lateral thinking (associative thinking affecting the way problems are perceived and structured) by DMs.

The CBR agent stores the decision process followed by a DM along with reflective observations on different aspects of the decision process and intermediate outcomes. While the DM is asked to fill in these reflective observations in the implemented agent, domain knowledge and user models can be incorporated into the agent to obtain these reflective observations autonomously. This stored decision process is replayed step by step by the CBR agent in the storyteller role. At each step the DM regains control of the system and is able to directly manipulate the decision situation and interact with the CBR agent. Through a "Next Step" button, the DM can require the agent to first comment on the event underlying the next state transition (e.g., "As a next step, $U$ introduced the criterion $C$ and assigned a $W$ importance to it" with $U=$ Peter, $C=$ "safety" and $W=$ high). The new state is then displayed on the screen with the related consequences for the MCDM problem considered in the case under consideration.

In the advisory role, the CBR agent attempts to exploit the knowledge stored in the case library to provide specific, context-related suggestions about problem solution. The CBR agent compares prior MCDM problem solutions with the current MCDM problem to offer specific suggestions such as "Why don't you consider $X$ as a relevant criterion?" or criticize choices made by the DM with statements like "Why do you assign so $\mathrm{much} / \mathrm{so}$ little importance to criterion $X$ ?" The DM can either ignore the advice or select a "Why" button to request an explanation of the CBR agent's rea- soning. The DM, stimulated by the explanation of the advice/suggestion, can also ask the CBR agent to display details from the relevant prior case.

The CBR procedure employed by the CBR agent is partially similar to the "monitor-analyze-advise" cycle of decision-aiding CB-DSSs such as CAL. However, the purpose underlying the cycle is different. The major goal of the CBR agent is to stimulate the DM into creative problem solution by enhancing learning both the decision process. The CBR agent exploits information related to decision processes within cases and other relevant knowledge to identify learning intervention conditions, i.e., stages in the decision process as which the DM needs to be told something or be stimulated into lateral thinking about some aspect of the decision process and/or outcome thus far. At each such learning intervention condition, the CBR agent utilizes its comparative knowledge about prior problem solutions in cases and the current problem (the nature of alternatives and criteria in a multicriteria decision-making problem) to offer specific context-related suggestions and/or criticisms.

As a final point, note that the CBR agent in Fig. 5 adapts its knowledge base by dynamically acquiring knowledge about the decision processes of the DM. This dynamic knowledge acquisition allows the CBR agent to incrementally customize its interactions with the DM.

\subsection{Summary}

The CBR procedure of conventional CB-DSSs as described in Fig. 3 can be seen to consist of three major parts: the case memory, the case retrieval procedure, and the case adaptation procedure. Different types of CB-DSSs tend to emphasize different aspects in each of these three components as summarized in Table 3.

The case memory in conventional CB-DSSs emphasizes the problem and solution descriptions in cases. The retrieval procedure matches current problem descriptions with case problem descriptions and the adaptation procedures adapt the retrieved case solution to the current problem.

Cases in decision-aiding CB-DSSs are moving toward emphasizing the decision process used to obtain the solution. The retrieval procedure is focused on the identification of appropriate decision and reflection-intervention features, i.e., aspects of the current decision process signaling that the DM needs help with the decision process. The DM is largely responsible for the adaptation procedures, i.e.,

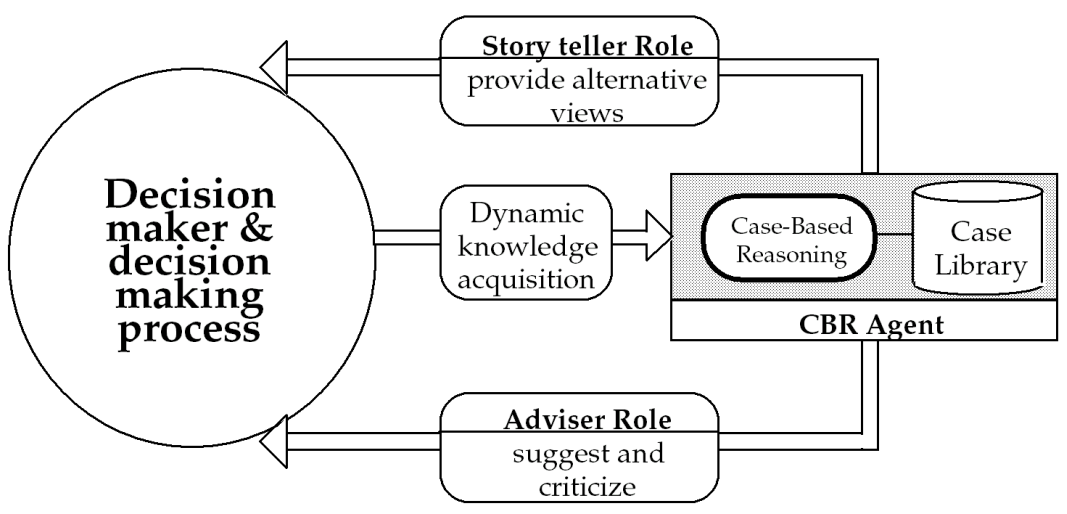

Fig. 5. Impact of the case-based reasoning agent on the decision-marking process. 
TABLE 3

Case-Based Reasoning in Different Case-Based Decision Support Systems

\begin{tabular}{|c|c|c|c|}
\hline & $\begin{array}{l}\text { Conventional CBR } \\
\text { Systems }\end{array}$ & $\begin{array}{l}\text { Decision-aiding CBR } \\
\text { Systems }\end{array}$ & Stimulative CBR Systems \\
\hline Case Memory & $\begin{array}{l}\text { Problem description } \\
\text { and solution to } \\
\text { problem }\end{array}$ & $\begin{array}{l}\text { Decision process used to } \\
\text { obtain solution to problem }\end{array}$ & $\begin{array}{l}\text { Learning-oriented reflec- } \\
\text { tive observations on deci- } \\
\text { sion process and interme- } \\
\text { diate solutions }\end{array}$ \\
\hline $\begin{array}{l}\text { Retrieval pro- } \\
\text { cedure }\end{array}$ & $\begin{array}{l}\text { Match current prob- } \\
\text { lem description with } \\
\text { case problem de- } \\
\text { scription }\end{array}$ & $\begin{array}{l}\text { - Identify decision inter- } \\
\text { vention features in current } \\
\text { decision process and } \\
\text { - Retrieve case which is } \\
\text { most relevant and useful } \\
\text { for guidance in current } \\
\text { decision process }\end{array}$ & $\begin{array}{l}\text { - Identify learning inter- } \\
\text { vention points in current } \\
\text { decision process and so- } \\
\text { lution obtained thus far; } \\
\text { and } \\
\text { - Retrieve case which is } \\
\text { most relevant for en- } \\
\text { hancing learning about } \\
\text { current decision process } \\
\text { and/or solution outcome }\end{array}$ \\
\hline $\begin{array}{l}\text { Adaptation } \\
\text { procedure }\end{array}$ & $\begin{array}{l}\text { Adapt case solution } \\
\text { to current problem }\end{array}$ & $\begin{array}{l}\text { - Decision-maker largely } \\
\text { responsible for adapta- } \\
\text { tion; and } \\
\text { - System may provide } \\
\text { some critiquing on deci- } \\
\text { sion process }\end{array}$ & $\begin{array}{l}\text { - Decision-maker respon- } \\
\text { sible for adaptation; and } \\
\text { - System supports active } \\
\text { experimentation by deci- } \\
\text { sion-maker }\end{array}$ \\
\hline
\end{tabular}

structuring the actual decision process. Depending upon the level of decision support offered, the CB-DSS may offer some critiquing with respect to the decision process being followed by the DM.

The evolving architecture of CBR in stimulative CB-DSSs is not clear given the limited current research in such systems. However, some initial hypotheses can be advanced. Cases in stimulative CB-DSSs would tend to emphasize both the decision process and the outcome with special emphases on aspects triggering learning intervention conditions. The retrieval procedure would focus on identifying appropriate learning-intervention features, i.e., stages in the decision process where the CB-DSS perceives a potential for the DM to enhance his/her learning about the decision process and the solution obtained thus far. The DM would again be largely responsible for the adaptation procedures, but the CB-DSS would tend to support active experimentation by the DM triggered by appropriate suggestions and/or criticisms.

\section{Contributions of Research}

This section compares and contrasts this paper with prior research and comments on its implications for current and future CBR research.

\subsection{Contributions Relative to Prior Research}

Research by psychologists and cognitive scientists has proven that humans routinely use CBR in their decision processes. According to experimental evidence [38], CBR is used by humans for a variety of tasks including learning new skills [53], [54], generating hypotheses about new situations/problems [41], [50], making decisions in complex and dynamic situations [33], explaining anomalous occurrences [49], and dealing with uncertain and incomplete information [34]. CBR researchers [36] have argued that CBR is useful both as a means for modeling human cognition and as a methodology for building intelligent systems. This has led to the significant interest in CBR research witnessed over the last decade.

While a variety of CBR systems have been designed and described in the literature, conceptual frameworks to ground the design and use of these systems have been conspicuously lacking. A common taxonomy has been to classify CBR systems functionally, i.e., according to the tasks for which they have been implemented such as planning [24], [25], design [28], diagnosis [40], interpretation [4], and classification [5]. An alternative classification [36] distinguishes between automated and interactive CBR systems. Two types of interactive systems have been identified:

- retrieval-only (which essentially act as smart retrieval systems), and

- advisory (which attempt to aid decision-making by a DM).

This research has utilized an integrative framework for the design of DSSs to identify three different types of CBR systems: conventional, decision-aiding and stimulative. The distinctions between these types of CBR systems are made along three dimensions: the object of decision support, the mode of decision support and emphasis of system design. Table 1 summarized the distinctive features of the three 
categories of CBR systems along the above dimensions. The key contributions of the proposed framework for CBR research and system design are described below.

First, the framework emphasizes the integration of CBR systems and DSSs. Such an emphasis is important because it provides a conceptual grounding for the emerging paradigm for the design and use of CBR systems as decision-aiding systems. The framework clarifies that all three types of CBR systems - conventional, decision-aiding, and stimulative-can be used for decision support, but each under specific organizational decision environments (Section 3.4). The purpose of decision support with each type of CB-DSS is different and these differences drive the design and implementation of the concerned CB-DSS (Section 4).

Second, the framework extends the emerging paradigm of decision-aiding CBR systems to identify stimulative CBR systems which aim to actively enhance learning in the DM about the decision situation. This is the synergistic result of mapping recent trends in DSS research onto CBR systems. For most of the past decade, the focus within CBR research has been on understanding the different facets of conventional CBR (Fig. 3). It is only recently, that the emphasis has explicitly started moving toward decision support. However, decision support is a rich topic which has been researched within the DSS community for the past two decades. The emphasis on learning-oriented DSSs is a current research focus within the DSS community. The framework surfaces the overall trend in the integration of CB-DSSs: from automation to decision-aiding and stimulation.

Third, the framework outlines important design implications for each type of CB-DSS. As the focus within conventional CB-DSSs is on the outcome object and automate mode of decision support, it is necessary to include all required knowledge to enable them to arrive autonomously at the right decision. A restrictive system design facilitates this automated production of the desired outcomes. The emphases within decision-aiding CB-DSSs is on the process object and informate mode of decision support. Thus it is necessary to extend the information content of cases to include detailed process knowledge and adapt the CBR procedures to exploit this decision process knowledge. The system design emphasis is no longer on knowledge for autonomous decision-making but on guiding the DM at appropriate points in the decision process. Stimulative CBDSSs focus on the learning object and stimulate mode of decision support. The system design emphasis is on knowledge for making suggestions/criticisms about both the decision process followed and the outcome obtained at any particular stage in the decision process. Such suggestions/criticisms need to be customized to the particular profile and needs of the DM.

Finally, the framework raises important research issues for the CBR community. Much of the research on conventional CB-DSSs has centered around the organization of case libraries, algorithms for retrieving cases and case adaptation procedures. The framework surfaces other research issues associated with decision-aiding and stimulative CB-DSSs. Decision-aiding CB-DSSs emphasize knowledge of the process of decision-making. This implies an extension in the information content of cases within conventional CB-DSSs. The impact of this expanded information within cases raises important questions about both case library representations and case retrieval algorithms. Retrieval algorithms now need to retrieve cases not solely based on initial problem or final solution descriptions, but need to account for aspects of the decision process which determine whether a case is relevant for decision support for the current decision process. Stimulative CB-DSSs raise an additional set of issues. The suggestions/criticisms of stimulative CB-DSSs are similar in nature to educational critiquing systems [15] but imply a further adaptation in the design of CBR procedures. The knowledge stored within cases should include models of the DM in addition to the decision process and case retrieval algorithms need to account for a different learning-oriented definition of relevance. To summarize, note that all aspects of CBR are different in the three types of CB-DSSs and these differences raise several open research issues for the CBR community.

\subsection{Conclusion}

CBR will remain a central component of artificial intelligence research due to its widespread usage by humans. However, the nature of CBR systems and research are evolving to make implemented CBR systems more relevant for real-life applications. Central to this evolution is the increased synergy between CBR systems and DSSs which several researchers have advocated recently [10], [20], [36], [38]. It is in the context of this evolution of CBR systems that the research presented in this paper is significant.

CBR systems are today making the shift from conventional to decision-aiding CB-DSSs. The framework presented in this paper has outlined a framework which provides a conceptual framework to guide this evolution and outlines the next challenge in this evolution-the move to stimulative CB-DSSs. All three types of CB-DSSs will be necessary, but the desired type of a CB-DSS will have to be adapted to the decision environment under consideration. The CBR research community will also need to tackle the newer set of research issues in CBR highlighted by this shift toward decision-aiding and stimulative CB-DSSs.

\section{REFERENCES}

[1] A.A. Angehrn and S. Dutta, "Case-Based Decision Support," Comm. ACM, to appear 1997-1998.

[2] A.A. Angehrn, "Stimulus Agents: An Alternative Framework for Computer-aided Decision-making," Proc. DSS 92, M.S. Silver, ed., Inst. of Management Science, pp. 81-92, June 1992.

[3] G. Ariav and M.J. Ginzberg, "DSS Design-A Systemic View of Decision Support," Comm. ACM, vol. 28, no. 10, pp. 1,045-1,052, Oct. 1985.

[4] K.D. Ashley, "Reasoning with Cases and Hypotheticals in Hypo," Int'l J. Man-Machine Studies, vol. 34, pp. 753-796, 1991.

[5] E.R. Bareiss, B.W. Porter, and C.C. Weir, "Protos: An ExemplarBased Learning Apprentice," Int'l J. Man-Machine Studies, vol. 29, pp. 549-561, 1988.

[6] R.H. Bonczek, C.W. Holsapple, and A.B. Whinston, Foundations of Decision Support Systems. New York: Academic Press, 1981.

[7] E. Domeshek and J. Kolodner, "Toward a Case-based Aid for Conceptual Design," Int'l J. Expert Systems, vol. 4, no. 2, pp. 201220, 1991.

[8] J.J. Donovan and S.E. Madnick, "Institutional and Ad-Hoc DSS and their Effective Use," Database, vol. 8, no. 3, pp. 79-88, Winter 1977. 
[9] J. Doyle, "A Truth Maintenance System," Artificial Intelligence, vol. 12, pp. 231-272, 1979.

[10] N.M. Duncan, "Case-Based Reasoning Applied to Decision Support Systems," masters thesis, Queen's Univ., Kingston, Canada, 1989.

[11] S. Dutta, Knowledge Processing and Applied Artificial Intelligence. Oxford, England: Butterworth-Heinemann, 1993.

[12] S. Dutta, "Using Case-based Reasoning for Supporting Decision Processes," INSEAD working paper 95/TM, 1995.

[13] S. Dutta, B. Wierenga, and A. Dalebout, "Designing Management Support Systems Using an Integrated Perspective," Comm. ACM, vol. 40, no. 6, pp. 70-79, June 1997.

[14] H.B. Eom, "The Current State of Multiple Criteria Decision Support Systems," Human Systems Management, vol. 8, pp. 13-119, 1989.

[15] G. Fischer, A.C. Lemke, T. Mastaglio, and A.I. Morch, "The Role of Critiquing in Cooperative Problem Solving," ACM Trans. Information Systems, vol. 9, no. 3., pp. 123-151, Apr. 1991.

[16] T.P. Gerrity Jr., "Design of Man-Machine Decision Systems: An Application to Portfolio Management," Sloan Management Rev., vol. 12, no. 2, pp. 59-75, Winter 1971.

[17] M.J. Ginzberg and E.A. Stohr, "Decision Support Systems: Issues and Perspectives," M.J. Ginzberg, W. Reitman and E.A. Stohr, eds., Decision Support Systems, North-Holland, Amsterdam, pp. 9-31, 1982.

[18] A. Goel and T. Callantine, "An Experience-Based Approach to Navigational Path Planning," Proc. IEEE/RSI Int'l Conf. Robotics and Systems, Raleigh, N.C., IEEE Press, New York, 1992.

[19] A. Goel, "Integration of Case-Based Reasoning and ModelBased Reasoning for Adaptive Design Problem Solving," PhD dissertation, Dept. of Computer and Information Science, Ohio State Univ., 1989.

[20] M. Goodman, "CBR in Battle Planning," Proc. DARPA Workshop Case-Based Reasoning, vol. 2, K. Hammond, ed., Morgan Kaufmann, San Mateo, Calif., pp. 246-269, 1989.

[21] M. Goodman, "Prism: A Case-Based Telex Classifier," Innovative Applications of Artificial Intelligence, vol. 2, A. Rappaport and R. Smith, eds., MIT Press, Cambridge, Mass., 1990.

[22] G.A. Gorry and M.S. Scott-Morton, "A Framework for Management Information Systems," Sloan Management Rev., vol. 13, no. 1, pp. 55-70, Fall 1971.

[23] R.D. Hackathorn and P.G.W. Keen, "Organizational Strategies for Personal Computing in Decision Support Systems," MIS Quarterly, vol. 5, no. 3, pp. 21-27, Sept. 1981.

[24] K. Hammond, Case-Based Planning: Viewing Planning as a Memory Task. Boston: Academic Press, 1989.

[25] K. Hammond, "CHEF: A Model of Case-Based Planning," Proc. AAAI 86, AAAI Press/MIT Press, Cambridge, Mass., 1986.

[26] J.C. Henderson and M.J. Martinko, "Cognitive Learning Theory and the Design of Decision Support Systems," Proc. DSS 81, First Int'l Conf. Decision Support Systems, Atlanta, pp. 45-50, 1981.

[27] D.H. Hennessy and D. Hinkle, "Applying Case-Based Reasoning to Autoclave Loading," IEEE Expert, vol. 7, no. 5, pp. 21-26, 1992.

[28] T.R. Hinrichs, Problem Solving in Open Worlds: A Case Study in Design. Northvale, N.J.: Erlbaum, 1992.

[29] R. Hogarth, Judgment and Choice: The Psychology of Decisions, New York: John Wiley \& Son, 1980.

[30] E.G., Hurst Jr., D.N. Ness, T.J. Gambino, and T.H. Johnson, "Growing DSS: A Flexible, Evolutionary Approach," J.L. Bennett, ed., Building Decision Support Systems, Addison-Wesley, Reading, Mass., pp. 111-132, 1983.

[31] S. Kambhampati and J.A. Hendler, "A Validation Structure Based Theory of Plan Modification and Reuse," Artificial Intelligence J., vol. 55, pp. 193-258, 1992.

[32] P.G.W. Keen, "Decision Support Systems: The Next Decade," Int'l J. Decision Support Systems, vol. 3, no. 3, pp. 253-265, 1987.

[33] G. Klein and R. Calderwood, "How Do People Use Analogues for Making Decisions?" Proc. DARPA Workshop Case-Based Reasoning, vol. 1, J. Kolodner, ed., Morgan Kaufmann, San Mateo, Calif., pp. 209-232, 1988

[34] G. Klein, L. Whitaker, and J. King, "Using Analogues to Predict and Plan," Proc. DARPA Workshop Case-Based Reasoning, vol. 1, J. Kolodner, ed., Morgan Kaufmann, San Mateo, Calif., pp. 224232, 1988

[35] D.A. Kolb, Experiential Learning. Engelwood Cliffs, N.J.: Prentice Hall, 1984
[36] J. Kolodner, Case-Based Reasoning. San Mateo, Calif.: Morgan Kaufmann, 1993

[37] J.L. Kolodner and R.L. Simpson, "The MEDIATOR: Analysis of an Early Case-Based Problem Solver," Cognitive Science, vol. 13, no. 4, pp. 507-549, 1989.

[38] J.L. Kolodner, "Improving Human Decision-making through Case-Based Decision Aiding," AI Magazine, pp. 52-68, Summer 1991.

[39] P. Korhonen, H. Moskowitz, and J. Wallenius, "Multiple Criteria Decision Support-A Review," European J. Operational Research, vol. 63, pp. 361-375, 1992.

[40] P. Koton, "Using Experience in Learning and Problem Solving," PhD dissertation, Dept. of Computer Science, MIT, 1989.

[41] J.S. Lancaster and J.L. Kolodner, "Varieties of Learning from Problem-Solving Experience," Proc. 10th Ann. Conf. Cognitive Science Soc., Lawrence Erlbaum, Hillsdale, N.J., 1988.

[42] D.B. Leake, Evaluating Explanations: A Content Theory. Northvale, N.J.: Erlbaum, 1992.

[43] D.B. Leake, "Goal-Based Explanation Evaluation," Cognitive Science, vol. 15, pp. 509-545, 1991.

[44] F.J. Lerch and M.M. Mantei, "A Framework for Computer Support in Managerial Decision-making," L. Maggi, J.L. King, and K.L. Kraemer, eds., Proc. Fifth Int'l Conf. Information Systems, Tucson, Ariz., pp. 129-1139, Nov. 1984.

[45] M.L. Manheim, "An Architecture for Active DSS," Proc. 21st Ann. Int'l Conf. System Sciences, IEEE CS Press, vol. 3, Hawaii, pp. 356$365,1988$.

[46] R.J. Mockler, Knowledge-Based Systems for Management Decisions, Prentice Hall, 1989.

[47] J.H. Moore and M.G. Chang, "Meta-Design Considerations in Building DSS," J.L. Bennett, ed., Building Decision Support Systems, Addison-Wesley, Reading, Mass., pp. 173-204, 1983.

[48] S.A. Raghavan and D.R. Chang, "Exploring Active Decision Support: The Janus Project," Proc. 22nd Ann. Hawaii Int'l Conf. System Sciences, pp. 33-45, 1989

[49] S. Read and I. Cesa, "This Reminds Me of the Time When ...: Expectation Failures in Reminding and Explanation," J. Experimental Social Psychology, vol. 26, 1990.

[50] M. Redmond, "Combining Case-Based Reasoning, ExplanationBased Learning, and Learning from Instruction," Proc. Sixth Int'l Workshop Machine Learning, S. Segre, ed., Morgan Kaufmann, San Mateo, Calif., 1989.

[51] E. Rich and K. Knight, Artificial Intelligence, Mc-Graw Hill, 1991.

[52] C.K. Riesbeck and R.C. Shank, Inside Case-Based Reasoning. N.J.: Lawrence Erlbaum Associates, 1989.

[53] B.H. Ross, "Remindings in Learning: Objects and Tools," Similarity and Analogical Reasoning, S. Vosniadou and A. Ortony, eds., Cambridge Univ. Press, New York, 1986.

[54] B.H. Ross, "Some Psychological Results on Case-Based Reasoning," Proc. DARPA Workshop Case-Based Reasoning, vol. 2, K. Hammond, ed., Morgan Kaufmann, San Mateo, Calif., pp. 144-147, 1989 .

[55] M.S. Silver, Systems that Support Decision-makers: Description and Analysis. John Wiley \& Son, 1991.

[56] S. Slade, "Case-Based Reasoning: A Research Paradigm," AI Magazine, pp. 42-55, Spring 1991.

[57] R.H. Sprague Jr. and E.D. Carlson, Building Effective Decision Support Systems. Englewood Cliffs, N.J.: Prentice Hall, 1982.

[58] C.B. Stabell, "A Decision-Oriented Approach to Building DSS," J.L. Bennett, ed., Building Decision Support Systems, AddisonWesley, Reading, Mass., pp. 221-260, 1983.

[59] E.P. Sycara, "Resolving Adversarial Conflicts: An Approach to Integrating Case-Based and Analytical Methods," Georgia Inst. of Technology, School of Information and Computer Science Technical Report No. GIT-ICS-87/26, Atlanta, 1987.

[60] E. Turban, Decision Support and Expert Systems. New York: MacMillan, 1990

[61] M. Zeleny, "Cognitive Equilibrium: A New Paradigm of Decision-Making?" Human Systems Management, vol. 8, pp. 185-188, 1989. 


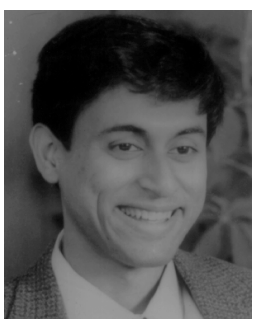

Soumitra Dutta obtained an MS degree in business administration and a PhD degree in computer science from the University of California at Berkeley. He is now an associate professor of technology management at the European Institute of Business Administration (INSEAD) in Fontainebleau, France. He is also a visiting associate professor at the Haas School of Business at the University of California at Berkeley. His research interests include the management of software development, knowledge-based systems, and the role of technology in business performance improvement initiatives. He is a member of the ACM, INFORMS, the IEEE, and the IEEE Computer Society.

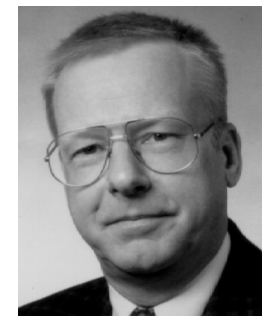

Berend Wierenga holds a PhD degree in marketing from Wageningen Agricultural University in The Netherlands. He is a professor of marketing at the Erasmus University in Rotterdam, Holland, and has held visiting positions at Stanford University, the Wharton School of the University of Pennsylvania, and the European Institute of Business Administration (INSEAD), Fontainebleau, France. His main research area is marketing management support systems. In this field, marketing models, econometric methods, and knowledge-based systems are used to support decision-making in marketing.

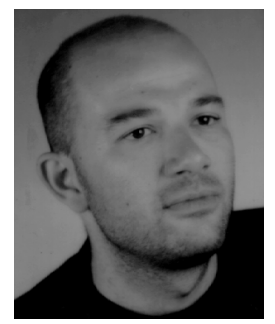

Arco Dalebout is a PhD student at the Erasmus University in Rotterdam, Holland, where he studies the design and use of software for marketing management support. His current project is the use of case-based reasoning systems for supporting sales promotion decision-making. 